\title{
Impact of Laser Energy on Synthesis of Iron Oxide Nanoparticles in Liquid Medium
}

\author{
Alemu Kebede ${ }^{1}$, Ashok V. Gholap ${ }^{1}$, Awadhesh K. Rai ${ }^{2 *}$ \\ ${ }^{1}$ Department of Physics, Addis Ababa University, Addis Ababa, Ethiopia \\ ${ }^{2}$ Laser Spectroscopy Research Laboratory, University of Allahabad, Allahabad, India \\ E-mail:nuuftoleeta@gmail.com, awadheshkrai@rediffmail.com \\ Received May 30, 2011; revised November 10, 2011; accepted November 20, 2011
}

\begin{abstract}
We present echofriendly laser ablation technique of Synthesizing iron oxide nanoparticle in pure water and discuss the impact of laser energy on the size, shape and morphology of the nanoparticle. The synthesized nanoparticle was characterized by UV/Visible absorption spectroscopy and morphological study was performed by scanning electron microscope (SEM). Intensity and wave length of the absorption peak of the colloidal nanoparticle prepared in water are dependent on the laser energy. Red-shift in the absorption band was observed at increasing laser energy. The intensity of absorption peak also changed when ablating laser energy was increased. The spherical natures of the nanoparticle is lost as the laser energy gradually increases and finally triangular shaped structures is observed as the laser energy increases from $9.3 \mathrm{~mJ}$ to $75 \mathrm{~mJ}$.
\end{abstract}

Keywords: Laser, Ablation, SEM, UV/Visible, Nanoparticle, Iron Oxide

\section{Introduction}

The electronic and optical properties and the chemical reactivity of small clusters/nanoparticles are completely different from the known property of each component in the bulk or at extended surfaces. The interaction forces crucially determine the properties of individual and collective nanoparticles. This interaction between nanoparticles resulting in aggregates may influence on their behavior [1]. Iron nanoparticles are of great interest due to their unique physicochemical properties and have been used for the development of imaging, purifying agents, magnetic and electrical applications. The structure and properties of the nanoparticle have been more investigated [1-6]. Much work on the synthesis of iron oxide nanoparticles have been done on bottom-up method in which chemicals are required for its synthesis [6,7]. But these methods are not echo friendly and may result in pollution of the environment. In addition to this there is possibility of the presence of impurities which create hindrance in using composites of the nanoparticle for medical purposes.

The laser ablation of a solid target immersed in a liquid environment has become an increasingly important top-down method for fabricating nano-structured materials $[8,9]$ which are free from chemicals. The physical approach was originally used to produce noble metal nanoparticles with bare surfaces, which cannot be achieved with wet chemical methods. Recently, Abhimanyu K. et al. [8] used pulsed laser ablation in liquid medium to synthesized and characterize noble metal nanoparticles. In this paper, we present an efficient and chemical-free method of preparation of colloidal iron oxide nanoparticle in water using laser ablation, and its characterization. The synthesized iron oxide colloidal nanoparticle loaded with insulin have been used for oral delivery in diabetic management.

\section{Experimental Details}

Iron slug (99.95\% pure), $9.5 \mathrm{~mm}$ dia $\times 9.5 \mathrm{~mm}$ length and $10 \mathrm{~g}$ mass was purchased from Alfa Aesar, USA. Ultra pure de-ionized water (HPLC grade) was purchased from Merck, Germany. Laser ablation of iron oxide nano-particle in pure water was performed by using Nd:YAG, pulsed laser (Continuum Surelite III-10, USA), using its second harmonic, (532 nm) wavelength, and having $4 \mathrm{~Hz}$ pulse repetition rate. The nanoparticle of iron oxide was synthesized at three different laser energies: $25.8 \mathrm{~m}, 75 \mathrm{~mJ}$ and $255 \mathrm{~mJ}$. A long focal length lens, $300 \mathrm{~mm}$ was use to focus the laser beam on to the material (Iron slug) emersed in $10 \mathrm{ml}$ ultra pure water. Abla- 
tion was made for 30 minutes. The experimental set-up for synthesis of iron oxide nanoparticle in water is shown in Figure 1.

\section{Results and Discussion}

Three different laser energies; $9.3 \mathrm{~mJ}, 25.8 \mathrm{~mJ}, 75 \mathrm{~mJ}$ at $4 \mathrm{~Hz}$ pulse repetition rate have been used for ablation purpose of iron slug immersing into $10 \mathrm{ml}$ pure water (Figure 1). The color of the solutions has gradually changed to dark brown (Figure 2) after ablation of the slug for 30 minutes which clearly indicates the formation of iron oxide colloidal nanoparticles.

UV/Visible spectroscopic technique has been used to verify the formation of iron oxide nanoparticles. The UV-Visible absorption spectrum of synthesized nanoparticle were recorded using Perkin Elmer Lambda-35 UV/ Vis spectrometer. Our experimental results clearly demonstrates that the position of absorption band and peaks (Figure 3) depends up on the laser energy which had been used to synthesize the iron oxide colloidal nanoparticles. The peak of the iron oxide colloidal nanoparticles shifted from $205 \mathrm{~nm}$ to $215 \mathrm{~nm}$ as the laser energy changed from $9.3 \mathrm{~mJ}$ to $75 \mathrm{~mJ}$ (Figure 3 and Table 1). The change in position of absorption peak of the iron oxide colloidal nanoparticles may be due to the change in the size of the colloidal nanoparticles. Our experimental results clearly reveales that smaller size nanoparticles are synthesized using lower laser energy $9.3 \mathrm{~mJ}$ (Figure 4(a)), and a tendency of agglomeration/cementation was also seen at higher laser energy $75 \mathrm{~mJ}$ (Figure 4(b)). This may cause a decrease in the reactivity of the nanoparticle as suggested by Paul G. et al. [10]. Further, it was observed tht the shape of the nanoparticles is changing with the laser energy, i.e., when the laser energy is increased the shape of the nanoparticlesit changes from spherical nature to triangular nature (Figure 4(c)). Therefore one may conclude that the spherical nanoparticles may be synthesized at lower laser energy. The other difficulty in synthesizing iron oxide nanoparticles at higher laser energy is associated with mechanical difficulties because because increasing laser energy resulted in the propagation of shock waves throughout the liquid medium and it is found difficult to control the rod at a fixed position during the ablation. At very high energy of the laser beam splashing of water from the container takes place and water from the container jumps out and collects at the surface of the focusing lens which prevents the transmission of the laser beam and finally stopped the ablation and this needs frequent cleaning of the focusing lens and there would also be loss of nanoparticles along with water splashing out of the container. The high local temperature of plasma during ablation of the nanoparti- cles in liquid solution may also have an impact on determining the optical properties of the nanoparticles.

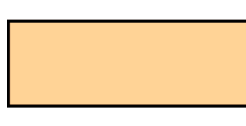

1

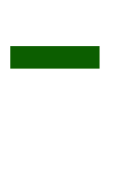

8

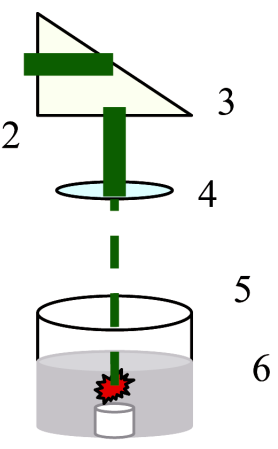

7
Figure 1. Experimental set up of laser ablation of iron oxide nanoparticle in water, 1: Laser source; 2: Laser light; 3: Right angled prism; 4: Converging lense; 5: Glass; 6: Water; 7: Iron slug; 8: Plasma.

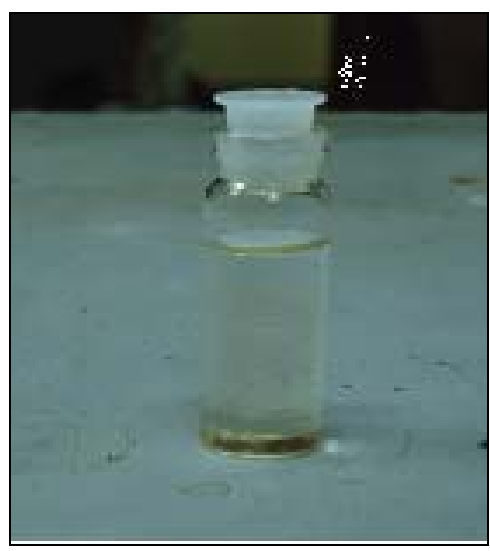

Figure 2. Iron oxide nanoparticle synthesized in water.

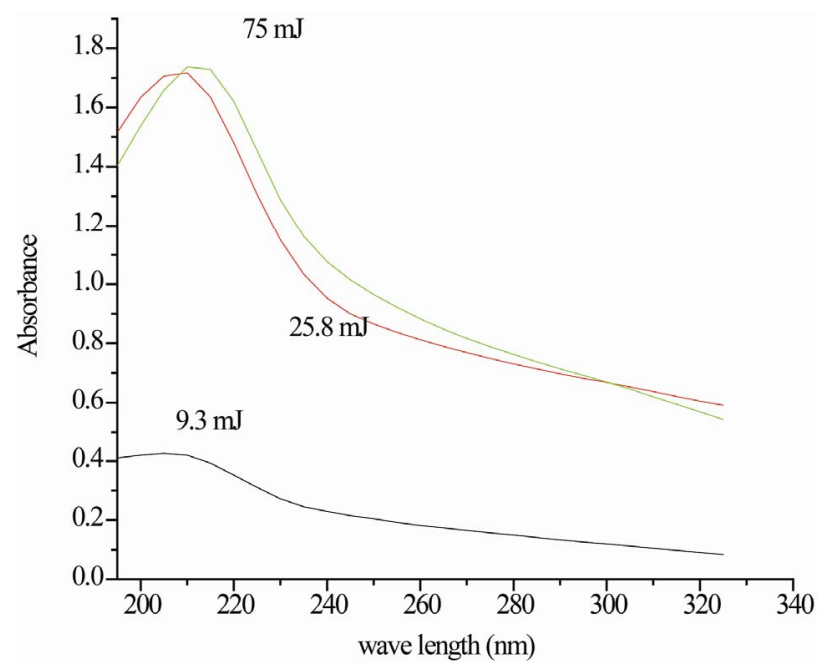

Figure 3. UV/Visible spectra of iron oxide nanoparticle in water. 


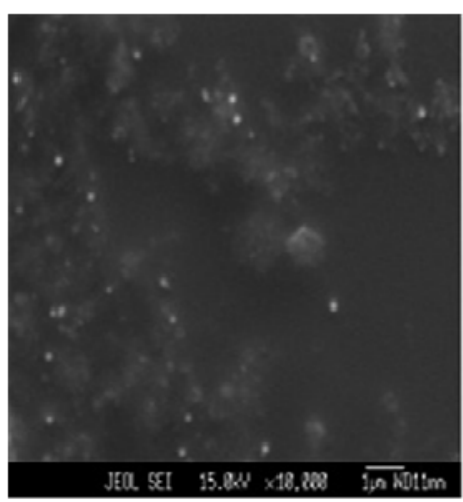

(a)

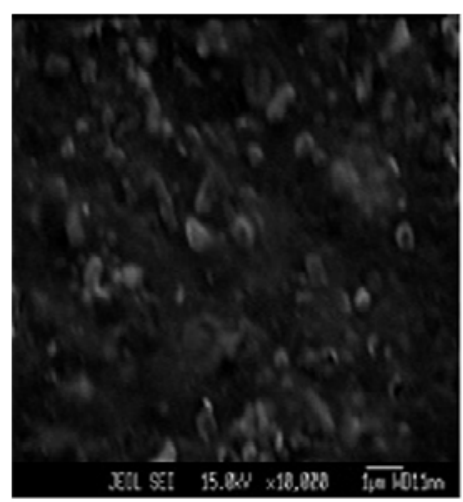

(b)

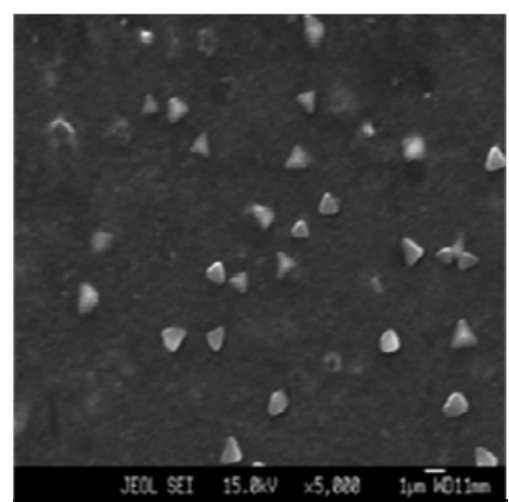

(c)

Figure 4. SEM image of colloidal iron oxide nanoparticles in water synthesized by laser ablation at (a) $25.8 \mathrm{~mJ}$; (b) cementation of nanoparticles $75 \mathrm{~mJ}$; (c) shape of nanoparticles (at the edge of a slide) at $75 \mathrm{~mJ}$.

Table 1. Dependence wave length and absorbance on laser energy.

\begin{tabular}{ccc}
\hline Absorbance & $\begin{array}{c}\text { Wave length } \\
(\mathbf{n m})\end{array}$ & $\begin{array}{c}\text { Laser energy } \\
(\mathbf{m J})\end{array}$ \\
\hline 0.425 & 205 & 9.3 \\
1.717 & 210 & 25 \\
1.729 & 215 & 75 \\
\hline
\end{tabular}

\section{Conclusions}

Iron oxide nanoparticles of different sizes and surface morphology have been successfully synthesized ultrapure water. The particle morphology showed dependence of shape and size of the nanoparticles on the laser energy which was further confirmed by the red shift in the absorbance peak.

\section{Acknowledgements}

I would like to acknowledge National Center for Experimental Mineralogy and Petrology, Allahabad University, for service they rendered me with scaning electron microscopic (SEM) image of the nanoparticles. I am thankful to Rohit Kumar, Abhimanyu K. Singh, Neeraj Giri and Ashok K. Pathak for their assistance and help. My special thanks goes to Prof. Ram Kripal for his assistance and provision of UV/Vis absorption spectrometer. Addis Ababa University, post graduate program, deserves acknowledgement for its financial assistance.

\section{References}

[1] Scientific Committee on Emerging and Newly Identified Health Risks (SCENIHR), "The Appropriateness of Existing Methodologies to Assess the Potential Risks Associated with Engineered and Adventitious Products of Nanotechnologies,” SCENIHR, 2006, p. 13.
[2] P. L. Apopa, Y. Qian, R. Shao, L. N. Guo, Diane Schwegler-Berry, M. Pacurari, D. Porter, X. L. Shi, V. Vallyathan, V. Castranova and C. D. Flynn, "Iron Oxide Nanoparticles Induce Human Microvascular Endothelial Cell Permeability through Reactive Oxygen Speceous Production and Microtubule Remodeling," Particle and Fibre Toxicology, Vol. 6, 2009, p. 1. doi:10.1186/1743-8977-6-1

[3] K.-C. Huang and K.-S. Chou, "Microstructure Changes to Iron Nanoparticles during Discharge/Charge Cycles,” Electrochemistry Communications, Vol. 9, 2007, pp. 19071912. doi:10.1016/j.elecom.2007.05.001

[4] H.-Y. Huang, Y.-T. Shieh, C.-M. Shieh and Y.-K. Twu, "Magnetic Chitosan/Iron (II, III) Oxide Nanoparticles Prepared by Spray-Drying,” Carbohydrate Polymers, Vol. 81, No. 4, 2010, pp. 906-910. doi:10.1016/j.carbpol.2010.04.003

[5] R. Singh, R. Verma, A. Kaushik, G. Sumana, S. Sood, R. K. Gupta and B. D. Malhotra, "Chitosan-Iron Oxide NanoComposite Platform for Mismatch-Discriminating DNA Hybridization for Neisseria gonorrhoeae Detection Causing Sexually Transmitted Disease,” Biosenceors and Bioelectronics, Vol. 26, No. 6, 2011, pp. 2967-2974.

[6] R. Sarkar, P. Pal, M. Mahato, T. Kamilya, A. Chaudhuri and G. B. Talapatra "On the Origin of Iron-Oxide Nanoparticle Formation Using Phospholipid Membrane Template,” Colloids and Surface B: Biointerface, Vol. 79, No. 12, 2010, pp. 384-389.

doi:10.1016/j.colsurfb.2010.04.023

[7] S. Laurent, D. Forge, M. Port, A. Roch, C. Robic, L. Vander Elst and N. Muller, "Magnetic Iron Oxide Nanoparticles: Synthesis, Stabilization, Vectorization Physicochemical Characterizations, and Biological Applications," Chemical Reviews, Vol. 108, No. 6, 2008, pp. 2064-2008. doi:10.1021/cr068445e

[8] A. K. Singh, A. K. Rai and D. Bicanic, "Controlled Synthesis and Optical Properties of Pure Gold Nanoparticles," Instrumentation Science and Technology, Vol. 37, No. 1, 2009, pp. 50-60.

[9] J. Perreire, E. Millon and E. Fogarassay (Eds.), "Recent Advances in Laser Processing of Materials,” Elsevier, Lon- 
don, 2006.

[10] P. G. Tratnyek, V. Sarathy and J. T. Nurmi, “Aging of Iron Nanoparticles in Water: Effects on Structure and Reactiv- ity,” Division of Environmental and Biomolecular Systems, Oregon Health and Science University, Portland, 2009. 